\title{
Ileal neuroendocrine tumor without metastatic disease presenting with chronic diarrhea
}

\author{
Jasmin Rahesh MS, MBA, Victor Mendiola BS, Baseer Quarashi BS, Srinivas Pathapati MD
}

\begin{abstract}
Neuroendocrine tumors in the gastrointestinal tract, formerly known as carcinoid tumors, are benign in presentation but can quickly metastasize if not diagnosed during regular screenings, such as colonoscopies. Unfortunately, most patients do not present for treatment until late with metastasis when symptoms become severe. We report a 65-year-old Caucasian man who presented with chronic diarrhea for over 2 months; this presentation suggested liver metastasis, but he had no metastasis on evaluation.
\end{abstract}

Keywords: Gastrointestinal carcinoid tumor, colonoscopy, ileal metastasis, neuroendocrine tumor

\section{INTRODUCTION}

Well-differentiated neuroendocrine tumors (NETs) are relatively rare tumors which commonly originate in the gastrointestinal tract, lung, and, rarely, the genitourinary tract. Forty-five percent of NETs originate in the small bowel, mainly the ileum, and present as a carcinoid syndrome. We report a case of ileal NETs presenting with chronic diarrhea as the only symptom without liver metastasis.

\section{CASE}

A 65-year-old Caucasian man with a medical history of hypertension and alcohol abuse presented to the emergency department with a 2-month history of watery diarrhea consisting of 3-4 episodes a day. He was transferred from an outside facility for acute renal failure, severe metabolic acidosis, diarrhea, dehydration, and hypokalemia. His initial episodes of watery diarrhea presented with fever, chills, and nausea. This

Corresponding author: Jasmin Rahesh

Contact Information: Jasmin.Rahesh@ttuhsc.edu

DOI: 10.12746/swrccc.v10i42.935 watery diarrhea increased to about 10 episodes a day during this initial course. He also reported generalized weakness and recurrent falls due to syncope. He self-medicated with Ivermectin in high doses used for horses that he bought from his hometown veterinarian with no prescription to treat his diarrhea and to prevent COVID-19 infections. He used this drug for 10 days and reported a 20-pound weight loss over 2 months and decreased urine output. During the time of this high-volume diarrhea, he also admitted to drinking about half a gallon of whiskey per week.

Following this initial course, his diarrhea continued at a lesser rate of only 3-4 episodes a day. However, three days prior to presenting to the emergency department he noticed fresh blood in his stool, prompting him to seek medical attention. On admission he denied abdominal pain, flushing, dyspnea, and chest pain. The patient denied any history of hepatitis, pancreatitis, peptic ulcer disease, or gastroesophageal reflux disease. He had never had a colonoscopy or endoscopy. On examination, he had mild diffuse abdominal tenderness.

On admission, his labs showed acute renal failure with a creatinine of $18 \mathrm{mg} / \mathrm{dL}$, a BUN of $161 \mathrm{mg} / \mathrm{dL}$, and bicarbonate of $6 \mathrm{mmol} / \mathrm{L}$. After he was started on fluid 


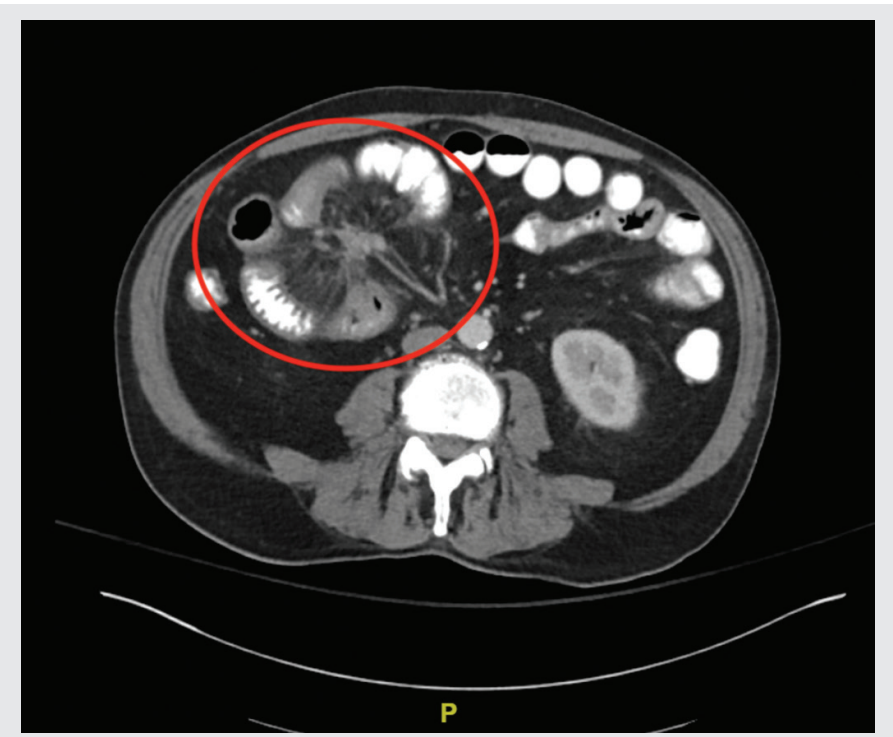

Figure 1. Computed tomography of the abdomen shows misty mesentery in the red circle.

resuscitation, his kidney function improved, his urinary output increased, and his acute kidney injury eventually resolved. Computed tomography (CT) of his abdomen was remarkable for "misty mesentery" suggestive of panniculitis (Figure 1). There was also enhancing nodularity of the distal small bowel mesentery in the right mid abdomen with mild to moderate circumferential bowel wall thickening of the underlying small bowel loops. There were scattered diverticula of the sigmoid colon with no diverticulitis. The carcinoid tumor was identified on the CT scan (Figure 2).

Colonoscopy showed a large $3 \mathrm{~cm}$ submucosal polypoid nodular mass $10 \mathrm{~cm}$ from ileocecal junction. Biopsy reported a well differentiated neuroendocrine tumor (carcinoid), at least $2 \mathrm{~mm}$ in depth with involvement of the lamina propria and submucosa (Figure 3). Liver ultrasound showed only hepatic steatosis with no lesions. A twenty-four-hour urine collection for 5-hydroxyindoleacetic acid (5-HIAA) was $12.5 \mu \mathrm{mol}$ (10.4-46.8 $\mu \mathrm{mol} / 24 \mathrm{~h}$ ); serum chromogranin A was $1048 \mathrm{ng} / \mathrm{ml}(21.0-108.0 \mathrm{ng} / \mathrm{mL}$, and serum serotonin was $883 \mathrm{ng} / \mathrm{ml}(101-283 \mathrm{ng} / \mathrm{mL})$. Transthoracic echocardiography showed no valvular abnormalities.

The right hemicolon with the small bowel mass was resected; surgical pathology reported a well

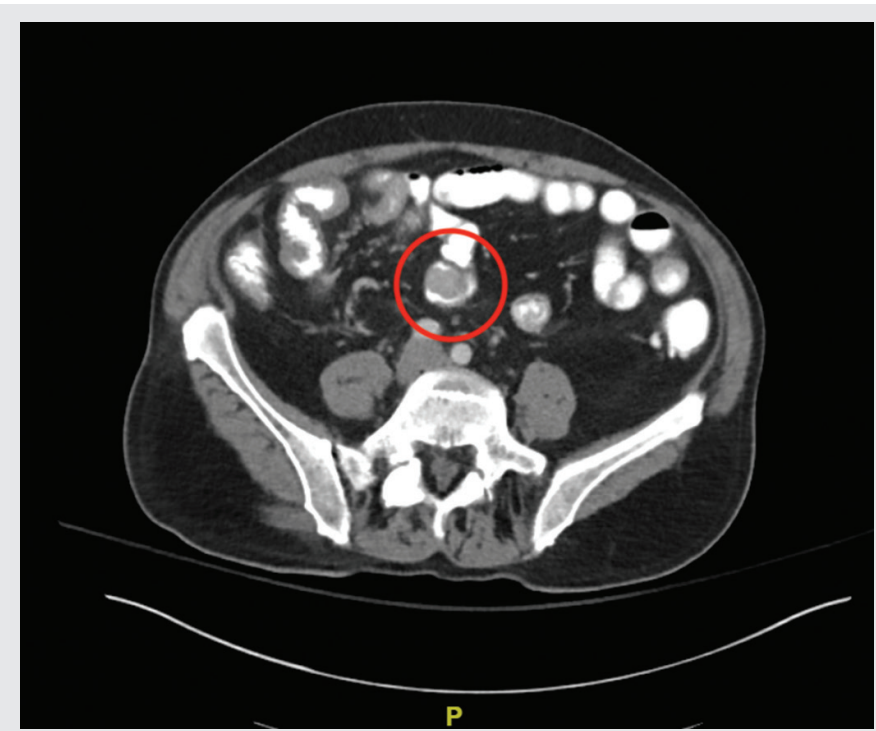

Figure 2. Computed tomography of the abdomen reveals the carcinoid tumor in the red circle.

differentiated neuroendocrine tumor, forming multiple (13) mucosal masses extending up to $2.3 \mathrm{~cm}$ in size with focal angiolymphatic invasion and $3 / 18$ regional lymph nodes involvement. After this surgery, his diarrhea improved, and patient followed up with a medical oncologist.

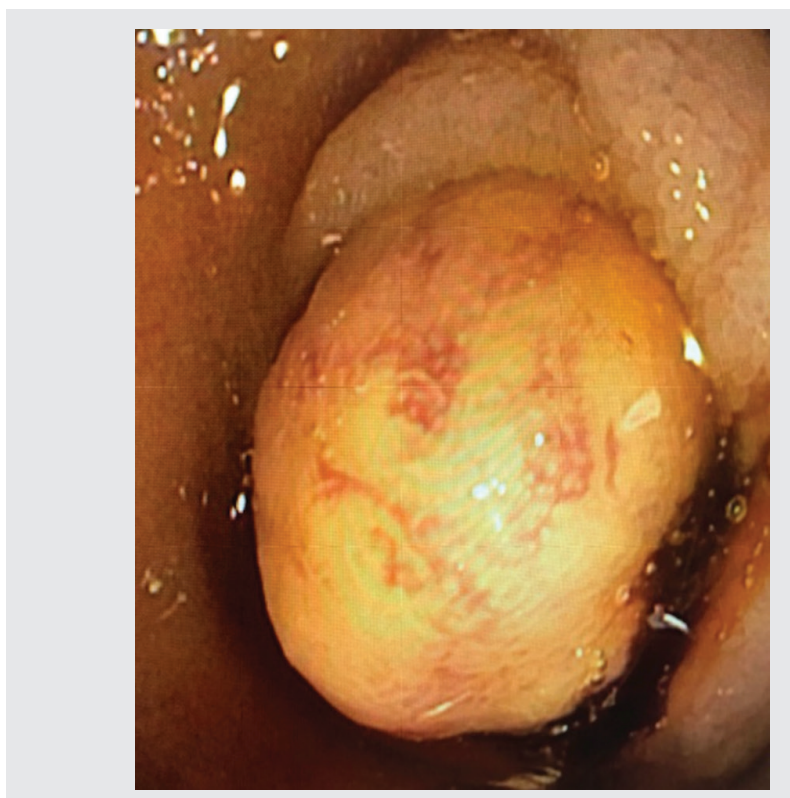

Figure 3. Carcinoid tumor identified on colonoscopy. 


\section{Discussion}

Neuroendocrine tumors most commonly develop in the gastrointestinal tract and were formerly named carcinoid tumors. These tumors arise from enterochromaffin cells of the digestive tract which contain serotonin. The World Health Organization distinguishes these tumors into well-differentiated and poorly differentiated categories. ${ }^{1}$

Small bowel neuroendocrine tumors like the one found in our patient are usually found incidentally and typically do not cause symptoms. However, if symptoms are present, it is commonly abdominal pain. Due to this seemingly innocuous presentation, these tumors are underdiagnosed and unrecognized. This is true especially in patients who do not receive recommended screenings with colonoscopies as in our patient. Often these tumors metastasize to the lymph nodes and liver because they are diagnosed in a late stage due to this benign symptom presentation. Tumors located in the jejunum and ileum often have a more aggressive clinical course. ${ }^{2}$

Lab values associated with neuroendocrine tumors include chromogranin, serotonin, and its end product 5-HIAA. Twenty-four-hour excretion of 5-HIAA has a high sensitivity and specificity for carcinoid syndrome. However, in our patient who presented with carcinoidlike symptoms of chronic diarrhea, these levels were normal at only $12.5 \mu \mathrm{mol} / 24$ hours. ${ }^{3}$ Although the 5-HIAA urine levels were normal, our patient had elevated serotonin and chromogranin markers indicating the need for more investigation. These markers are considered less reliable in literature reports and frequently represent false positive test results, but they were the only indicators of gastrointestinal tumor in our patient.

After tumor metastasize, more symptoms arise which are characteristic of carcinoid syndrome and include flushing and diarrhea. ${ }^{4}$ Although these symptoms are usually associated with a late-stage tumor, our patient had characteristic chronic diarrhea but presented with no metastasis. The recommended treatment for tumors which have not metastasized is a resection of the bowel and mesentery, which resolved our patient's symptoms. ${ }^{5}$

In summary, this case focuses on chronic diarrhea being the sole presenting symptom in ileal carcinoid tumor without objective evidence of metastasis.

Article citation: Rahesh J, Mendiola V, Quarashi B, Pathapati S. Ileal neuroendocrine tumor without metastatic disease presenting chronic diarrhea. The Southwest Respiratory and Critical Care Chronicles 2022;10(42): 25-27

From: Department of Internal Medicine, Texas Tech University Health Sciences Center, Amarillo, Texas

Submitted: $12 / 20 / 2021$

Accepted: 1/9/2022

Conflicts of interest: none

This work is licensed under a Creative Commons Attribution-ShareAlike 4.0 International License.

\section{REFERENCES}

1. Rindi G, Klimstra DS, Abedi-Ardekani B, et al. A common classification framework for neuroendocrine neoplasms: an International Agency for Research on Cancer (IARC) and World Health Organization (WHO) expert consensus proposal. Mod Pathol 2018;31(12):1770-1786.

2. Burke AP, Thomas RM, Elsayed AM, et al. Carcinoids of the jejunum and ileum: an immunohistochemical and clinicopathologic study of 167 cases. Cancer 1997;79(6):1086-1093.

3. Sjöblom SM. Clinical presentation and prognosis of gastrointestinal carcinoid tumours. Scand J Gastroenterol 1988;23(7): 779-787.

4. Saha S, Hoda S, Godfrey R, et al. Carcinoid tumors of the gastrointestinal tract: a 44-year experience. South Med J 1989; 82(12):1501-1505.

5. Pape UF, Perren A, Niederle B, et al. ENETS Consensus Guidelines for the management of patients with neuroendocrine neoplasms from the jejuno-ileum and the appendix including goblet cell carcinomas. Neuroendocrinology 2012;95(2): $135-156$. 